\title{
El rol del espacio público en el desarrollo urbano resiliente desde una perspectiva de la niñez: el caso de los cerros de Valparaíso, Chile
}

\section{The role of public space in resilient urban development from a childhood perspective: The case of the Valparaíso's Hills, Chile}

\author{
Marcela Soto Caro ${ }^{1}$ \\ ORCID: http://orcid.org/0000-0003-3566-2349 \\ Correo electrónico: marcela.soto@usm.cl
}

\author{
Anne Escobar Guéguen ${ }^{1}$ \\ ORCID: http://orcid.org/0000-0002-0548-2067
}

\begin{abstract}
${ }^{1}$ Departamento de Arquitectura, Universidad Técnica Santa María de Valparaíso, Valparaíso, Chile.
Esta investigación fue apoyada por la Dirección de Investigación, Innovación y Postgrado de la Universidad Técnica Federico Santa María, como parte del proyecto de investigación Los nuevos roles del espacio público en la construcción y educación de la civilidad y los riesgos de origen antrópico o natural: un enfoque en la niñez. Proyecto Interno Línea de Investigación USM Los Nuevos Roles del Espacio Público AR4005.
\end{abstract}

\section{Resumen}

El espacio público juega un rol esencial dentro del desarrollo urbano resiliente; su cualidad de espacio abierto y su capacidad social de albergar a la población son dos de sus características más importantes. A pesar de lo anterior, el espacio público y su funcionalidad generalmente no están considerados en la planificación y mitigación ante desastres socio-naturales. En este caso la población infantil, ciertamente una de las más vulnerables, es prácticamente invisible en medio de las medidas de mitigación y reconstrucción post catástrofe. Dado lo anterior, esta investigación busca avanzar en la comprensión de las percepciones de los niños y niñas sobre el espacio público, centrándose en el papel que podría desempeñar en el desarrollo urbano resiliente. Para ello, se utilizó un enfoque comparativo, basado en tres espacios públicos ubicados en los cerros de la ciudad de Valparaíso, un territorio caracterizado por su vulnerabilidad a incendios y terremotos. La metodología consistió en la realización de grupos focales y talleres creativos con niños y niñas que estudian en establecimientos educacionales cercanos a estos espacios. Finalmente, los resultados evidencian que en aquellos espacios públicos donde se utilizó metodologías de diseño participativo, los niños y niñas son más propensos a entenderlos como refugios ante siniestros.

Palabras clave

Desarrollo urbano resiliente; desastres socio-naturales; espacio público; niñez; territorios vulnerables

\begin{abstract}
Public space plays an essential role in resilient urban development. The open space it provides and the ability to accommodate the population are two of their most crucial characteristics in this respect. Despite the preceding, public space and its functionality amid socio-natural disasters are usually not considered in risk planning and management. The child population, certainly among the most vulnerable, is equally invisible amid mitigation measures and postcatastrophe reconstruction. Thus, this article seeks to advance the understanding of children's perceptions of public space, focusing on the role it could play in resilient urban development. To this end, a comparative approach was used, based on three public areas located in the hills of the city of Valparaíso, a territory characterized by its vulnerability to urban fires and earthquakes. The methodology consisted of focus groups activities besides using creative, art-based methods to engage children in participatory workshops. Finally, the results show that in those public spaces where participatory design methodologies were used, children are more likely to understand them as shelters from socio-natural disasters.
\end{abstract}

\section{Keywords}

Childhood; public space; socio-natural disasters; urban resilience development; vulnerable territories 


\section{Introducción}

Actualmente existe una fuerte tendencia a nivel mundial a que los niños abandonen el espacio público para irse al espacio privado (Gülgönen \& Corona, 2015; Ridgers, Knowles \& Sayers, 2012). En comparación con la década de 1970 en Latino América, se ha constatado una reducción significativa del tiempo que un niño destina a actividades al aire libre correspondiente a un 50\% (Juster, Stafford y Ono, 2004). Por ejemplo, en Chile un niño pasa más del $60 \%$ de su tiempo en espacios cerrados y poco ventilados como casas, jardines y escuelas (Ministerio de Salud, 2009). Diversos son los factores y razones por las que los niños y niñas rechazan usar el espacio público, como la peligrosidad, la basura, la contaminación, la falta de higiene, la infraestructura deteriorada, entre otros (Churchman, 2003; Rubio y Santarelli, 2011). Lo anterior estaría provocando, entre otras cosas, el desconocimiento de los beneficios que su entorno les pudiese brindar a ellos y sus familias (Consejo Nacional de la Infancia, 2016), esencialmente el rol que podría jugar como espacio de protección y albergue frente a desastres socio-naturales. De esta manera, tenemos una deuda como sociedad en visibilizar las percepciones de los niños y niñas ante desastres socio-naturales y estudiar con detención los efectos adversos que generen en su población, sobretodo aquellas más vulnerables.

En contextos de desastres socio-naturales, la población infantil destaca por presentar mayores grados de vulnerabilidad que el resto de la población (Carr, Abad Merchán, \& Ullauri, 2020). Los niños corren un mayor riesgo de morir o sufrir lesiones, de contraer enfermedades potencialmente mortales y de ser desplazados en situaciones de desastre porque generalmente son más débiles, menos móviles, tienen sistemas inmunológicos inmaduros y no tienen control directo sobre el medio ambiente en el que viven (Peek, 2008). Su bienestar depende del cuidado, protección y de las oportunidades que le entreguen sus familias, comunidad y de la sociedad en general (Carr et al, 2020; Bourke, 2014). Sin embargo, sus necesidades y preferencias muchas veces son omitidas en las decisiones que afectan a su entorno físico, natural y social (Bourke, 2014; Harden, Backett-Milburn, Scott
\& Jackson, 2000; Valentine, 2004). Según el informe Social protection for children at times of disaster (CEPAL, 2017), a pesar que la frecuencia de los desastres en la región latinoamericana ha aumentado 3,6 veces entre 1960 y 2010, no existe información sobre la población infantil afectada. Sin embargo, dos tendencias sugieren que el número de niños y niñas afectados por desastres es elevado y creciente. Por una parte, el aumento del número de desastres $\mathrm{y}$, por la otra, el crecimiento de la población infantil (CEPAL, 2017).

En virtud de lo anterior, la presente investigación busca avanzar en la percepción que tienen los niños y niñas sobre el espacio público, especialmente ante el rol que pudiera jugar como espacio seguro, ante los riesgos de origen antrópico o natural. Para ello, se trabajó a partir de tres casos de estudios correspondientes a tres espacios públicos situados en los cerros de la ciudad de Valparaíso territorio que, además de tener un alto déficit de espacios públicos y áreas verdes, se caracteriza por su vulnerabilidad frente a la propagación de incendios urbanos y sismos. Los tres casos de estudio fueron seleccionados de acuerdo al grado de inclusión de la población infantil tanto en el diseño como en el equipamiento existente en ellos referidos al disfrute de la población infantil. En este respecto, se consideró un primer espacio diseñado con la participación activa de niños y niñas; otro diseñado sin su participación pero que contempla una cancha y juegos infantiles; $y$ un tercer espacio el cual su diseño no ha sido vinculado a la niñez, ni tampoco contiene elementos para el disfrute de esta población. Esta diferenciación nos permite aventurarnos en plantear la siguiente hipótesis: dado que los espacios públicos diseñados con la participación activa de la población infantil tienden a ser más cercanos para los niños y niñas, pudiera existir una actitud más positiva hacia éstos como espacios de albergue y refugio ante siniestros socio-naturales.

La metodología plantea la utilización de técnicas cualitativas como grupos focales y talleres creativos para el estudio de las opiniones y actitudes de niñas y niños pertenecientes a establecimientos educacionales aledaños a los espacios públicos seleccionados. De esta manera, temas referentes al uso y apreciación del espacio público y de su rol ante un desarrollo urbano resiliente 
fueron abordados en diversas actividades con el objetivo de obtener la percepción de una población vulnerable y silente, pero muy necesaria: la población infantil.

El artículo se organiza de la siguiente manera: la primera sección presenta el marco teórico dividido en dos subsecciones. La primera subsección plantea la relación actual existente entre el espacio público y el desarrollo urbano resiliente. La segunda subsección plantea la necesidad de incorporar a la población infantil niñez en temas de planificación y diseño urbano, titulado una perspectiva desde la niñez. En esta última además se agrega otro apartado que refiere a la incorporación también de la niñez, pero específicamente ligado a la planificación de un desarrollo urbano resiliente. La segunda sección presenta los casos de estudio, referidos a los tres espacios públicos seleccionados, incluida una descripción de Valparaíso y su condición de vulnerabilidad tanto social como territorial ante riesgos de origen antrópico y natural. Luego, la tercera sección presenta la metodología, dividida en 3 procesos metodológicos. El primero, referido a la realización de grupos focales, el segundo a un taller, mientras que en el tercero se presentan los protocolos realizados para el trabajo en conjunto a la población infantil. Una cuarta sección presenta los resultados con respecto a las percepciones de esta población ante los espacios públicos en evaluación recabadas en las actividades realizadas. Una vez obtenidos los resultados, se presenta una quinta sección correspondiente a la discusión. Finalmente se presenta una sexta y última sección que contiene las conclusiones del estudio y termina por generar una reflexión en torno al rol actual del espacio público.

\section{Marco Teórico}

\section{Espacio Público y el Desarrollo Urbano Resiliente}

La creciente concentración de población, bienes y activos en aglomeraciones urbanas, sumado al aumento de la frecuencia de desastres socio-naturales como terremotos, tsunamis o incendios, ha puesto en la palestra la vulnerabilidad de los asentamientos humanos. Estos desastres han llevado a repensar las ciudades y a desarrollar y fomentar la capacidad de estas para afrontar situaciones adversas con el fin de salvaguardar tanto a la población que las habita como su desarrollo (Sharifi \& Yamagata, 2014). Lo anterior está estrechamente relacionado con el concepto de desarrollo urbano resiliente, que ha ido ganando relevancia rápidamente en la literatura sobre planificación y la sostenibilidad urbana.

De esta manera, el desarrollo urbano resiliente puede definirse como la capacidad que tiene una ciudad para resistir, adaptarse y recuperarse de manera oportuna y eficaz ante amenazas como catástrofes socio-naturales (Allan, Bryant, Wirsching, García \& Rodríguez, 2013). Desde un principio, los esfuerzos para aumentar la resiliencia de las ciudades se basaron en factores económicos, políticos, sociológicos, o visiones culturales (Montejano y Moreno, 2016). Sin embargo, en los últimos tiempos, el espacio público se ha incorporado como un sistema capaz de reducir y mitigar riesgos, pudiendo a la vez contribuir al proceso de recuperación de una ciudad (Allan et al., 2013; French, Birchall, Landman, \& Brown, 2019; Koren \& Rus, 2019).

Registros históricos de eventos sísmicos en todo el mundo reafirman la importancia de estos espacios debido a su capacidad para acomodar y albergar a la población durante y después de eventos catastróficos (Godschalk, 2003; Koren \& Rus, 2019). Por ejemplo, en períodos post catástrofe, estos espacios funcionan como verdaderas centrales para la gestión de actividades cotidianas como vivienda, salud, distribución de bienes y servicios, eliminación de residuos, e incluso comercio (Middleton, 2007). Dado lo anterior, es que se ha reconocido mundialmente que el espacio público podría ser tremendamente útil para hacer frente a las amenazas socio-naturales. Tal como se concertó en la Nueva Agenda Urbana ${ }^{1}$ con respecto al espacio público, se prevén espacios articuladores del tejido urbano que, además de ser inclusivos, de calidad, abiertos, seguros, accesibles, verdes y multifuncionales,

1 Documento resultante del acuerdo en la conferencia de Hábitat III realizada en Quito año 2016 que funciona como guía para orientar los esfuerzos en materia de desarrollo de las ciudades. 
éstos deben poder incrementar la resiliencia de las ciudades frente a desastres socio-naturales (Naciones Unidas, 2016).

Otros investigadores sostienen que las ciudades no sólo logran un desarrollo urbano resiliente a través de gestión y planificación del riesgo de desastres, sino que las redes sociales y culturales dentro de una comunidad también son sumamente importantes (Campanella, 2006; Derr, Corona, \& Gülgönen, 2019; Fletcher, Cox, Scannell, Heykoop, Tobin-Gurley, \& Peek, 2016). La resiliencia urbana no implica una única reconstrucción del hábitat; la población afectada también puede acudir a los contextos sociales los que pueden brindar apoyo a personas y a satisfacer sus necesidades, ayudando a su recuperación. En este sentido, el espacio público también ha sido ampliamente reconocido por su calidad para crear, mejorar y fortalecer los vínculos sociales dentro de un territorio (Borja y Muxi, 2000; Valentine, 2004). Así, el espacio público podría jugar otros roles para contribuir a la planificación y gestión de riesgos, como proporcionar una plataforma capaz de fortalecer las redes e identidades sociales y culturales, fomentando la resiliencia. A pesar de lo anterior, el espacio público y su funcionalidad ante desastres socio-naturales no suelen considerarse en la planificación y gestión de riesgo (Berroeta, Carvalho \& Di Masso, 2016).

\section{Una perspectiva desde la niñez}

Un creciente interés por estudiar la participación de la población infantil en temas de planificación y diseño urbano se ha hecho notar. En las últimas décadas, diversas investigaciones en todo el mundo han planteado que la planificación urbana, incluido el diseño de espacios públicos, tienden a ser más exitosos con la participación directa de la población infantil (Bishop \& Corkery, 2017; Cele \& van der Burgt, 2015; Derr, 2015; Driskell, 2002; Freeman, 2006; Simpson, 1997). Asimismo, indican que la participación comunitaria activa de los niños puede mejorar sustancialmente la calidad de vida, y no sólo para la población infantil, sino para todos los integrantes de una comunidad (Brown et al., 2019; Freeman, Henderson, \& Kettle,1999; Freeman \& Tranter, 2011). Esta visión se sustenta en el principio que los niños pueden ser generadores de vida comunitaria, insinuando que la presencia de niños puede influir positivamente en la percepción de las personas sobre el espacio público y motivarlas a pasar más tiempo en él (Unicef, 2014). En este sentido, los niños pueden convertirse en agentes atractores, proporcionando oportunidades para pasar más tiempo al aire libre e incentivando la interacción social entre personas de todas las edades. Dado lo anterior, existe un emergente y fuerte llamado a todas las ciudades a ser ciudades amigas de la infancia ${ }^{2}$ (Unicef, 2014), que sugiere que "si podemos construir una ciudad exitosa para niños, será una ciudad exitosa para todos" (Enrique Peñalosa, citado en Gilbert \& O'Brien, 2005, p. 5).

En muchos sentidos, las características de las ciudades amigas de la infancia son también las que harán que nuestras ciudades sean más resilientes (Derr, Chawla, \& Van Vliet, 2017; Freeman \& Tranter, 2011; Tranter \& Malone, 2008). La resiliencia, entendida como la capacidad de adaptarse a las amenazas externas, también es evidente cuando los niños exhiben fortalezas al buscar atención y apoyo, y cuando las personas y los lugares que los rodean puedan proporcionarles el apoyo y los recursos que ellos necesitan (Derr et al., 2017). En este sentido, y como se mencionó anteriormente, el espacio público también puede jugar un papel fundamental en el desarrollo de la resiliencia, en cuanto a proporcionar un entorno adecuado para reproducción social, incluido el apoyo emocional de los niños (Derr et. al., 2019).

A pesar de lo anterior, los niños han sido tradicionalmente incomprendidos y obviados como participantes activos en sus comunidades (Amri, Haynes, Bird, \& Ronan, 2018; Bourke, 2014). Oportunidades para que los niños den a conocer sus opiniones sobre los problemas de sus

2 Una Ciudad Amiga de la Infancia es cualquier ciudad, pueblo, comunidad o sistema de gobierno local comprometido con el cumplimiento de los derechos de las niñas, los niños y los adolescentes de acuerdo con la Convención sobre los Derechos del Niño (ciudadesamigas.org). 
vecindarios y comunidades, incluso en lo que respecta a las medidas de mitigación y de reconstrucción postcatástrofes, son prácticamente inexistentes.

Desarrollo Urbano Resiliente y Niñez. Históricamente, los niños han sido poco estudiados en la planificación urbana resiliente y excluidos principalmente en la práctica (Amri et al., 2018). Una ausencia absoluta de la participación de este grupo etario en el marco de políticas públicas y programas dirigidos al hábitat comunitario, así como en las medidas destinadas a promover la resiliencia, la mitigación y la reconstrucción post desastres socio-naturales se ha constatado (Carr et al., 2020; Fletcher et. al, 2016; Fundación Supera la Pobreza, 2016). Sin embargo, en los últimos años un creciente cuerpo literario sugiere incluir a los niños como participantes activos en los procesos de planificación de la resiliencia urbana dado que se ha demostrado que su participación puede mejorar y reforzar este tipo de desarrollo (Derr et al., 2019; Fletcher et al., 2016; Freeman \& Tranter, 2011). Centrarse en salvaguardar los derechos del niño en la planificación de la resiliencia urbana puede ampliar la conceptualización del bienestar en ciudades resilientes (Derr et al., 2019). A este respecto, se reconoce que las ciudades amigas de la infancia podrían empoderar los procesos de guiar a las ciudades hacia un desarrollo urbano resiliente, haciéndolas más saludables, más habitables y sostenibles y no solo para los niños sino para todos los habitantes de la ciudad. Crear comunidades capaces de salvaguardar los derechos del niño a la protección, provisión, y participación (Unicef, 2014) conducirá a niños y ciudades más resilientes (Freeman \& Tranter, 2011).

\section{Casos de estudio}

La presente investigación compara tres casos de estudio correspondientes a tres espacios públicos ubicados en los cerros de la ciudad-puerto de Valparaíso. Capital de la Provincia y Región homónimas, Valparaíso se ubica sobre la costa del Océano Pacífico, a $110 \mathrm{~km}$ al oeste de Santiago, capital de Chile. Con una densidad poblacional de 737,9 hab $/ \mathrm{km}^{2}$ (Instituto Nacional de Estadísticas, 2017), es centro histórico del área metropolitana de Valparaíso. Única por su topografía, los componentes geográficos que caracterizan su conformación urbana y paisajística son: el sector plan $^{3}$ (referido a la planicie costera), sus cerros, los que se encuentran distribuidos en arco de poniente a oriente y la bahía conformada por los dos anteriores como tercer componente.

Asociado íntimamente a su geografía, características climatológicas, territoriales e incluso sociales, Valparaíso se enfrenta frecuentemente a determinados riesgos que pueden a su vez llegar a convertirse en desastres socio-naturales. Entre ellos, los riesgos más relevantes son el riesgo a incendio y a sismos (Municipalidad de Valparaíso, 2010). Respecto a este último, Chile es uno de los países más sísmicos del mundo ${ }^{4}$ lo cual refleja una amenaza constante tanto para la población de Valparaíso como para el resto del país. En cuanto al riesgo a incendio, Valparaíso se ubica en torno a un sector con daño potencial alto por incendios forestales donde los mayores riesgos de esta índole se localizan hacia la periferia de la ciudad en zonas donde el bosque exótico se introduce y donde además se ubica la mayor cantidad asentamientos de carácter informal (PNUD, 2014). Esta forma irregular de habitar el territorio fomenta las condiciones necesarias para la generación de incendios. La localización de asentamientos en quebradas, la generación de basurales, entre otras, han contribuido a que estos riesgos se conviertan en verdaderas catástrofes, como ocurrió con el mega incendio de Valparaíso del año 2014, considerado el incendio urbano más grande del país (MINVU, 2015).

Gran parte de la ciudad presenta un hábitat precario en una accidentada geografía de cerros y quebradas con altos índices de vulnerabilidad que exacerban los riesgos a

3 El 'plan' es la forma popular de denominar la estrecha planicie costera existente entre la bahía y sus cerros.

4 Después de Japón, Chile es el segundo país más sísmico del mundo y el cuarto que arriesga mayores daños por catástrofes naturales, según el reporte de la Organización para la Naciones Unidas (ONU) en la III Conferencia Mundial de Reducción del Riesgo de Desastres, realizada en marzo 2015 en Sendai- Japón (Naciones Unidas, 2015). 




Fuente: Elaboración propia.

la que la ciudad se encuentra expuesta. De esta manera los cerros de Valparaíso se convierten en un territorio interesante de abordar en esta investigación ya que presentan en su conjunto, un escenario de restricciones socioeconómicas y amenazas ambientales que afectan a la población infantil con mayor intensidad.

\section{Espacios Públicos Seleccionados}

Como se mencionó anteriormente, la selección de los espacios públicos a evaluar se realizó según el grado de inclusión de la población infantil en su metodología de diseño. De esta manera se seleccionó una primera plaza ubicada en cerro Cárcel llamada Bismark (1) la cual carece de elementos para el disfrute de la población infantil. Luego, una segunda plaza ubicada en cerro Monjas llamada Esmeralda (2), fue seleccionada, la cual contempla la instalación de una serie de juegos infantiles, máquinas para hacer ejercicio y una multicancha. A pesar de contener diversos elementos para el disfrute de la población infantil, esta no fue partícipe del proceso de diseño de este espacio. Finalmente, la tercera plaza seleccionada se encuentra ubicada en las alturas del cerro Placeres, llamada Esperanza Viva (3). Dicha plaza es un espacio recuperado por organizaciones sociales $\mathrm{y}$ vecinos que decidieron transformar un sitio eriazo en un espacio para el disfrute de toda la población. Aquí la metodología de diseño consistió en la inclusión de la población infantil durante todo el proceso, tanto en el diseño como en su ejecución (Fundación Mi Parque, 2016). Una vez seleccionados los espacios públicos, se localizaron los establecimientos educacionales cercanos a estos, los que serán identificados como: EE1 y EE2 cercanos a plaza Bismark, EE3 cercano a plaza Esmeralda y EE4 cercano a plaza Esperanza Viva (Ver Figura 1, 2 y Tabla 1).

\section{Metodología}

El proceso metodológico contempló la identificación, comparación y una evaluación in-situ de los tres espacios públicos seleccionados según en el grado de inclusión de la población infantil en la metodología de diseño de cada espacio. Esta selección nos permitirá comparar las actitudes y percepciones de los niños hacia cada plaza para verificar si los espacios públicos diseñados con la participación activa de los niños son más exitosos que aquellos que se han diseñado sin su participación (Bishop 
Figura 2

Espacios públicos seleccionados



Fuente: Elaboración propia.

Tabla 1

Descripción de los Espacios Públicos seleccionados.

\begin{tabular}{|c|c|c|c|c|}
\hline Espacio Público & Equipamiento & Creación & Metodología de Diseño & Grupos participantes \\
\hline Plaza Bismark & $\begin{array}{l}\text { Máquinas de ejercicio. } \\
\text { Pequeño anfiteatro. } \\
\text { Mirador. }\end{array}$ & $\begin{array}{l}\text { Data de finales del } \\
\text { siglo XVIII con menores } \\
\text { renovaciones desde } \\
\text { entonces. }\end{array}$ & $\begin{array}{l}\text { Sin la participación active } \\
\text { de la población infantil. }\end{array}$ & $\begin{array}{l}\text { Grupos } 1 \text { y } 2 \\
\text { pertenecientes a EE1 y } \\
\text { EE2 respetivamente. }\end{array}$ \\
\hline Plaza Esmeralda & $\begin{array}{l}\text { Juegos Infantiles. } \\
\text { Multicancha. } \\
\text { Pequeño anfiteatro. }\end{array}$ & $\begin{array}{l}\text { Remodelada por programa } \\
\text { gubernamental "Quiero Mi } \\
\text { Barrio" en el año } 2010 .\end{array}$ & $\begin{array}{l}\text { Sin la participación active } \\
\text { de la población infantil. }\end{array}$ & $\begin{array}{l}\text { Grupos } 3 \text { y } 4 \\
\text { pertenecientes a EE3. }\end{array}$ \\
\hline Plaza Esperanza Viva & $\begin{array}{l}\text { Juegos Infantiles. } \\
\text { "trunks." } \\
\text { Cajas de arena. }\end{array}$ & $\begin{array}{l}\text { Creada por una asociación } \\
\text { de ONG en el año } 2016 \text {. }\end{array}$ & $\begin{array}{l}\text { Con la participación active } \\
\text { de la población infantil. }\end{array}$ & $\begin{array}{l}\text { Grupos } 5 \text { y } 6 \\
\text { pertenecientes a EE4 }\end{array}$ \\
\hline
\end{tabular}

Fuente: Elaboración propia.

\& Corkery, 2017; Driskell, 2002; Freeman, 2006; Simpson, 1997). En ese sentido, se podría asumir que este éxito debiera reflejarse en un mayor sentido de apropiación del espacio público y, por tanto, en una actitud más positiva en cuanto a si el espacio sería útil como refugio frente a siniestros. Para ello, se identificaron establecimientos educacionales cercanos a estos espacios con la finalidad de tener acceso a una población infantil que estudie en las inmediaciones de estos.

Una vez identificada y caracterizada la población a estudiar, se elaboró un formato que combinó la realización de un focus group y un taller. La base teórica para formular el primer instrumento radica en la obtención de información cualitativa mediante discusiones orientadas a indagar acerca de actitudes y reacciones de los niños y niñas con respecto al espacio público (Edmunds, 1999; Morgan, Gibbs, Maxwell, \& Britten, 2002). A su vez, con el objetivo de poder cristalizar las ideas del grupo, la actividad se complementó con un taller en el cual los niños realizaron dibujos con respecto al tema planteado, además de la realización de intervenciones en una maqueta de cada plaza. De esta manera, se propone finalizar el trabajo con niños y niñas mediante una instancia lúdica y creativa, resultando además un mecanismo que permitirá ordenar la información y visualizar las expectativas de este grupo. 


\section{Grupos Focales}

Para la realización de las actividades tipo focus group se determinó formar seis grupos (dos referentes a cada plaza), cada uno con $n=10$ niñas y niños, mediante una selección aleatoria simple. Dicha selección se realizó con el apoyo institucional de los establecimientos, luego de contactar a los respectivos directores de cada colegio. Para conformar grupos representativos se siguieron los siguientes criterios: representatividad en los rangos de edad de niñas y niñas, de 8 a 11 años; número similar de niñas y niños en cada grupo; que habiten en distintas áreas de la comunidad y con distintos perfiles académicos.

Cada una de las actividades fue llevada a cabo en los respectivos establecimientos, dirigida por una psicóloga y presenciada por el equipo investigador y personal de cada institución. El registro de los grupos focales se realizó a través de grabaciones en audio y video, cuyo contenido se transcribió posteriormente. De manera de salvaguardar la identidad de los participantes se asignó un código a cada uno, para luego pedirles indicar edad y dirección. Este último con la intención de conocer distrito en el que viven y según eso conocer el grupo socioeconómico (GSE) al que pertenecen.

Las actividades consistieron en reunir estos grupos de niños y niñas con el fin de tener una conversación inicial respecto a la plaza correspondiente en cada caso, y explicando al grupo en qué consiste la investigación y la ubicación de esta. De esta manera, se realizó en primera instancia una actividad expositiva/conversacional con foco introductorio al tema de los espacios públicos, para luego efectuar la lluvia de ideas y conversación respecto a las expectativas de cada grupo, la cual culminó con una actividad de intervención, el taller.

\section{El Taller}

La actividad culminó con el taller donde los niños y niñas participantes de la actividad pudieron generar de manera lúdica propuestas de diseños para sus respectivas plazas como también indicar aquellos espacios que consideran de mayor importancia dentro de estas. Así, en una primera instancia, se les hizo entrega a cada niño y niña, una hoja de block en blanco además de una variedad de lápices de colores. Luego, se les indicó a los niños que pudieran dibujar libremente en base a la conversación mantenida durante el grupo focal. Una vez realizados los dibujos, se les presentó una maqueta de cada plaza, tridimensional y en blanco, realizada por el equipo de investigación. Una vez que los niños visualizaron la maqueta y lograron ubicarse en el espacio, se les pidió que expresaran sus ideas, recomendaciones y propuestas para cada espacio. A medida que las ideas por parte del grupo iban surgiendo, un integrante del equipo de investigación iba anotando las ideas en post-its de distintos colores y ubicándolos en los lugares señalados por los mismos niños. De esta manera, se realizaron intervenciones en la maqueta con respecto a propuestas de diseño para sus respectivas plazas, así como también, con respecto a los espacios que consideraban más importantes y de refugio ante siniestros.

\section{Protocolo}

Antes de realizar las actividades, se hizo entrega de un primer documento informado sobre la investigación a cada uno de los establecimientos educacionales, el cual fue firmado por ambas partes (directores y los profesionales a cargo de la investigación). Luego, se les hizo entrega de un oficio de autorización a los apoderados de cada uno de los niños y niñas seleccionados para la actividad. En este oficio se detalló, indicando fecha y hora de su realización, de qué se trata la investigación y la descripción de la actividad incluyendo la captura audiovisual de esta. Una vez obtenido el consentimiento de padres, apoderados y de autoridades de los colegios, mediante estos documentos firmados por cada uno de los involucrados, se procedió a realizar las actividades.

\section{Resultados}

Con una cantidad total de 62 niños participantes, entre ellos 33 hombres y 29 mujeres, la edad promedio de estos fue de 10 años. En cuanto a su caracterización socioeconómica predominan los alumnos cuyos hogares se encuentran en distritos donde prima el GSE "E" 
correspondiente a un 50\% de los participantes. Así mismo ocurre con todos los grupos a excepción del grupo 2 en que predomina uno de los más altos, el GSE "C2" correspondiente al EE2.

\section{Resultados de los Grupos Focales}

En cuanto a la realización de la actividad tipo focus group, los resultados se presentan mediante las siguientes categorías: (1) reconocimiento de la plaza, (2) si hacen uso de la plaza, (3) con quién la visitan, (4) actividades que realizan en la plaza, (5) cómo se sienten cuando están en la plaza y (6) utilidad ante desastres socio-naturales. A continuación, para la identificación de los participantes se utilizará la sigla Gn1_n2, donde n1 será el número del grupo correspondiente y $\mathrm{n} 2$, al respectivo número asignado a cada uno de los participantes.

1. Todos los grupos reconocen inmediatamente sus respectivas plazas. Sin embrago existe una diferencia en la actitud que adoptan al mostrar la imagen de las plazas, donde los niños pertenecientes a EE3 (grupos 3 y 4) y a EE4 (5 y 6) son los que demuestran mayor sentido de aprecio y reconocimiento de plaza como también su nombre. Sin embargo, en el caso de los alumnos del EE4, su plaza llamada Esperanza Viva, es más conocida como la plaza del "Santa Isabel" una cadena de supermercados que aportó con el financiamiento de esta plaza. “¡Esa es nuestra plaza! ¡Esa de ahí!” (G3_5). “¡La plaza Esmeralda!” (G3_10).

“Es la plaza! ¡la plaza Esmeralda!” (G4_4).

“¡Es la plaza de aquí!” (G5_2).

“¡Es la plaza del Santa Isabel!” (G6_5).

2. Si bien la mayoría de los que estudian en cercanías a la plaza Bismark afirman ir a la plaza, hubo casos que dijeron ir poco e incluso no ir de manera tajante. En caso contrario, los alumnos pertenecientes a EE3 (grupos 3 y 4) por unanimidad manifiestan frecuentar la plaza de manera constante. Cabe mencionar el hecho que el EE3 contempla la plaza para sus actividades curriculares como talleres de medio ambiente y educación física. En el caso del
EE4, al igual que el anterior, manifiestan visitar de manera continua la plaza, sin embargo, hubo un par de niños que dice no ir muy a menudo ya que residen en sectores más alejados.

3. En esta sección, al igual que en la anterior, vuelve a ser importante la acción del EE3 de potenciar un espacio público de cercanía. Esto en primer lugar porque no se observa autonomía por parte del grupo etario por lo tanto su posibilidad de acceder a estos espacios está sujeta en primer lugar a la disposición de un familiar. Así lo expresó la mayoría del grupo de la plaza Bismark, y de la plaza Esperanza Viva mientras que en los grupos de la plaza Esmeralda la participación del profesor es de mayor importancia.

4. En todos los grupos el juego es primordial. Es la primera respuesta que surge de manera inmediata y espontánea. Los grupos 3 y 4 de la plaza Esmeralda, le dan un uso excepcionalmente recreativo-deportivo; los grupos 5 y 6 de la plaza Esperanza Viva le dan un uso recreativo donde prima el juego colectivo; y por último algunos alumnos de los grupos 1 y 2 ven a la plaza Bismark sólo de manera funcional, como por ejemplo ir a tomar locomoción.

"Yo sólo voy a tomar la micro ahí" (G1_8).

"Yo voy a jugar a los juegos de "atrás” (G1_3).

Mientras que G1_2 le responde a G1_3: "Ahí no hay juegos, sólo máquinas de hacer ejercicio”.

5. Para todos los grupos las plazas cercanas representan un lugar donde experimentan sentimientos de felicidad y agrado. Sin embargo, surgen algunos casos puntuales que refieren a indiferencia o a sentirse asustado. En este último caso basado en experiencias vividas dentro de las plazas.

"Una vez vi a una señora que le estaba pegando a su hija por no sé, la niña estaba puro diciendo garabatos y casi la empuja y casi se cae” (G2_10).

6. En los grupos de la plaza Bismark (1 y 2) no hay total claridad, diferenciándose estrechamente el grupo 2 del 1 por una actitud más positiva hacia el rol de la plaza ante un sismo o un incendio (Figura 3). Así 



Fuente: Elaboración propia.

también los grupos de la plaza Esperanza Viva (5 y 6) manifiestan sus diferencias, sin poder llegar a un consenso sobre esta. Por otra parte, los alumnos del EE3 sí creen por unanimidad que la plaza es útil en caso de sismo y están divididos 50/50 en el caso que la plaza sea útil ante un incendio.

En términos generales, la mayoría de los niños participantes tienen una actitud positiva hacia las plazas en caso de sismo en gran parte por representar un espacio abierto. Sin embargo, comentarios como "habría que pensar bien...hay unos pilares que pueden caer" (G2_5) surgen haciendo alusión a la peligrosidad de elementos verticales dentro de la plaza. Mientras que las opiniones sobre la utilidad de la plaza ante un incendio están divididas. En este último las razones radican en que hay mucha vegetación y puede hacer que el fuego se propague con más fuerza, o bien, por la inexistencia de agua, mangueras, grifos, etc.

\section{Resultados del Taller}

En la primera actividad del taller, los niños tuvieron la libertad de dibujar sus percepciones y aprensiones con respecto a cada plaza según las conversaciones mantenidas en los grupos focales anteriores. En esta sección, se destacaron las ideas relacionadas con las preocupaciones de los niños por la amenaza de incendios, así como también se recopilaron algunas ideas interesantes sobre tener cuerpos de agua cerca de los espacios públicos para combatir estas amenazas (Figura 4).

En cuanto a la segunda actividad del taller correspondiente a las intervenciones en la maqueta tridimensional, todos los niños respondieron bien a lo que se les estaba preguntando y aportaron con diversas ideas. Los dos grupos que trabajaron sobre la plaza Bismark (1 y 2) coincidieron en nombrar como zonas de importancia a los árboles, las máquinas de hacer ejercicio (las que ocupan como juego ya que la plaza carece de juegos infantiles), el mirador y lugar donde tomar la locomoción. En cuanto a lo que cambiarían o agregarían a la plaza, coinciden plenamente en agregar juegos infantiles. Luego las ideas son diversas, siendo el grupo 2, pertenecientes a EE2 quienes sugieren mayores cambios y con ideas más creativas como casa en el árbol, luces en el suelo, fuente de agua, casa para perros, entre otros. Así también este grupo hizo menciones sobre la seguridad de la plaza como poner paso peatonal, paradero para la locomoción, poner guardia, entre otros. Lo anterior se puede atribuir al modelo educativo que emplea el EE2 llamado “constructivista”, el cual es enfático en el desarrollo artístico integral del estudiante y en la educación medioambiental y patrimonial.

Los grupos 3 y 4 pertenecientes a EE3 coinciden plenamente en que los lugares de mayor relevancia son la cancha, los juegos y los árboles. En cuanto a qué cambiarían y agregarían, coinciden en hacer reparos a la cancha, 


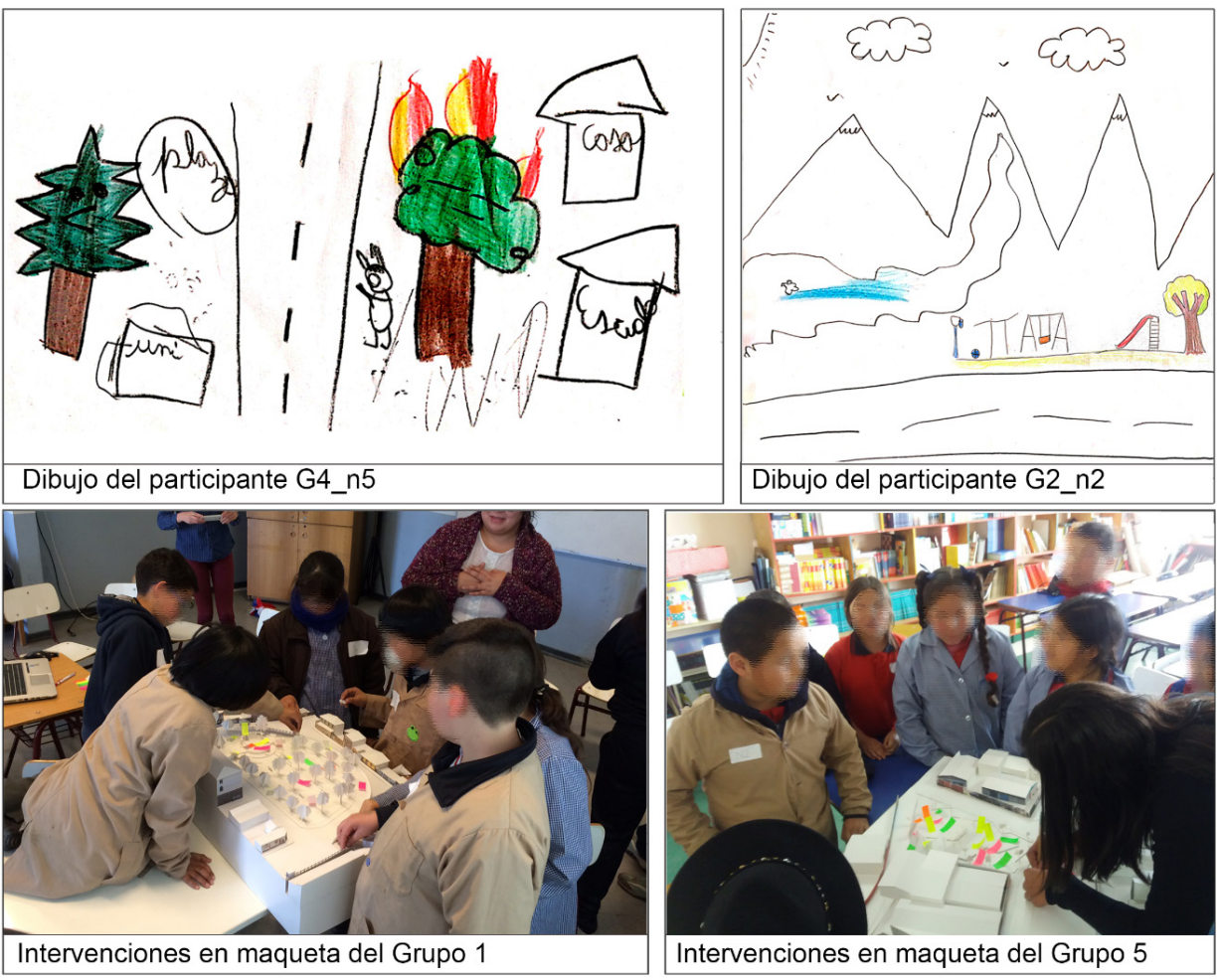

Fuente: Elaboración propia.

a poner más sombras y a acciones que resguarden su seguridad ante el tránsito vehicular como poner semáforos, pasos peatonales, entre otros. Cabe mencionar que estos dos grupos que trabajaron sobre la plaza Esmeralda tienen pleno conocimiento de ella, ambos grupos de la plaza Esmeralda reconocen y especifican los lugares con total concordancia entre ellos. De manera contraria, los dos primeros grupos no reconocieron completo equipamiento de la plaza Bismark. En este último hay discusiones con respecto al mobiliario existente, por ejemplo, algunos llaman a las máquinas de ejercicios existentes como "los juegos” y otros corrigen que son máquinas.

Los grupos 5 y 6 coinciden en cuanto a los lugares más importantes dentro de la plaza Esperanza Viva, difiriendo en uno o dos elementos. Aquí resalta la metodología utilizada en el diseño de esta plaza, puesto que los niños coinciden en dar importancia a los elementos que fueron diseñados con y para ellos como un arenero y los "troncos" refiriéndose a una intervención realizada en madera para el disfrute infantil. Así también sus propuestas contemplan aprovechar del territorio como por ejemplo toboganes en la pendiente y miradores sobre sombreadores.

\section{Discusión}

Si bien la intención de comparar los tres casos de estudio refiere a que el sentimiento de apropiación del niño(a) hacia un espacio público estaría sujeto a los procesos de diseño de las plazas estudiadas, la investigación demuestra que los modelos educativos de los establecimientos educacionales resultan ser aún más relevantes en cuanto a facilitar al niño(a) la interacción e integración en su hábitat comunitario. Como era de esperar, la plaza Bismark (que no incluye a esta población en su diseño), no hace parte importante de la vida diaria y comunitaria de muchos de los niños que estudian en sus inmediaciones, sin embargo, un grupo en particular reconoce grandes potenciales en ella. Nos referimos al grupo 2, perteneciente al EE2 que, a pesar de no hacer uso de la plaza en su malla curricular, este establecimiento ofrece un modelo educativo distinto que aborda el desarrollo artístico integral del estudiante y su educación medioambiental y patrimonial. Como resultado, dicho grupo fue el que contribuyó con mayores ideas, y siendo también más creativos en sus propuestas de intervención y diseño; por ejemplo, el dibujo elaborado por G2_n2 expuesto en la Figura 4 que refiere a la existencia 
de cuerpos de agua cercanos a la plaza de manera de hacer frente a amenazas como incendios.

Por otra parte, los grupos de las plazas Esmeralda y Esperanza Viva, ambas contenedoras de equipamiento para el disfrute de la población infantil, demostraron mayor aprecio y conocimiento de estas. A pesar de lo anterior, hubo algunas diferencias entre ambos casos de estudio. Si bien el diseño de la plaza Esmeralda contempla a la población infantil, -pero sin incluirla en el proceso de diseño como lo hace la plaza Esperanza Viva- los grupos de esta primera plaza demostraron mayor conocimiento de esta y una actitud más positiva en cuanto a su rol protector ante un siniestro. Lo anterior es atribuible también al modelo educativo del establecimiento educacional que, integrando este espacio público en su malla curricular, logra brindar a esta población la posibilidad de generar una mayor apropiación sobre este y de interactuar con su hábitat comunitario de manera constante.

Lo anterior cobra aún más relevancia al tratarse de una población infantil que pertenece a grupos socioeconómicos más vulnerables, que no cuentan con un espacio público seguro cercano a sus hogares, como manifestó en su gran mayoría la población infantil perteneciente al EE3 (pertenecientes al GSE “D”). De esta manera, tanto el diseño como los modelos educativos de los establecimientos educacionales estarían incidiendo en el aprecio y sentido de apropiación que tienen los niños hacia estos espacios como también en la percepción de los roles que el espacio público pudiera brindar en un desarrollo urbano resiliente.

Cabe mencionar, que siendo pobladores de un hábitat vulnerable a desastres socio-naturales como terremotos e incendios, los niños manifestaron mayores preocupaciones en cuanto al tráfico vehicular. Esta preocupación puede estar asociada al hecho que el tráfico vehicular es una amenaza más común con la cual deben lidiar a diario, y que además lo reconocen como una situación que puede mejorar para ellos mediante la realización ciertas intervenciones, como cerrar calles, poner lomos de toro, incluir semáforos, entre otros.

\section{Conclusiones}

Según los resultados arrojados en esta investigación, la hipótesis en un principio planteada es corroborada. Efectivamente, existe una mayor aprobación en cuanto al uso de espacio público como refugio ante siniestros de parte de niños y niñas de las plazas, donde se utilizó una metodología de diseño participativa con la población infantil. Sin embargo, otro factor igualmente incidente en sus percepciones fue identificado; nos referimos a los modelos educativos de los establecimientos educacionales. El caso correspondiente al EE3, que contiene dentro de su malla curricular el uso activo y continuo de estos espacios, les facilita la interacción e integración de los niñas y niños con su hábitat comunitario; y con ello, una actitud más positiva ante el rol que el espacio público pudiera desempeñar ante siniestros.

De esta manera podemos concluir que el espacio público puede asumir un rol protector ante siniestros sólo sí se generan buenas experiencias para los niños en estos espacios. Si niñas y niños no tienen oportunidad de vivir y apropiarse de espacios públicos, a través de actividades propias de su edad relacionadas fundamentalmente con el juego, es difícil que reconozcan y que aprecien la función individual y social que el espacio público les puede brindar. Así mismo es fundamental que conozcan y se familiaricen con estos espacios y sus entornos. Por lo anterior, tanto el rol de la población en el diseño como el fomento de la utilización de estos espacios por parte de las autoridades es fundamental. Al tratarse de una población con baja autonomía, el uso activo de los espacios públicos está sumamente ligado en una primera instancia, al núcleo familiar como agente promotor del uso del espacio público. Sin embargo, muchas veces, y por diversas causas, la promoción del uso del espacio público por parte de un familiar no siempre es certera. En tales situaciones la acción institucional y comunitaria cobra mayor importancia para la adaptación y apreciación del niño(a) de su hábitat en pos al fomento de una cultura de seguridad y resiliencia, mediante la utilización del conocimiento, la innovación y la educación. 
Siendo el caso de estudio los cerros de Valparaíso, un hábitat en que catástrofes socio-naturales son frecuentes como terremotos e incendios, la población infantil bajo estudio demostró tener nociones básicas de qué hacer frente a este tipo de siniestros (como, por ejemplo, resguardarse en los cerros ante un terremoto y posible tsunami). Tal característica está inserta en la cultura colectiva incluyendo la población infantil, sin embargo, el espacio público y su rol ante este tipo de situaciones no está integrado de la misma manera, al menos no en esta población. En este sentido, el espacio público no estaría considerando las condiciones propias de la ciudad ni tampoco su propio potencial en temas de resiliencia urbana. Por ello se sugiere, a partir de las discusiones generadas tras esta investigación, que espacios públicos como las plazas Bismark, Esmeralda y Esperanza Viva puedan contar con información sobre cómo protegerse ante estos siniestros, tener señalamientos explícitos sobre zonas de seguridad a su interior, llevar a cabo revisiones de su infraestructura y áreas para certificar que brinden protección (podría considerarse además la realización de simulacros periódicos de emergencia para instruir a la población escolar acerca de los uso de estos espacios). Todo esto pone de manifiesto que el diseño del espacio público se debe reflexionar en un contexto amplio de necesidades sociales, condiciones y perfil de la ciudad y que esta es una reflexión y estrategia que aún falta por desarrollar en términos teóricos y prácticos.

\section{Referencias bibliográficas}

Allan, P., Bryant, M., Wirsching, C., García, D., \& Rodríguez, M. (2013). The Influence of Urban Morphology on the Resilience of Cities Following an Earthquake. Journal of Urban Design, 18(2), 242-262. https://doi.org/10.108 0/13574809.2013.772881

Amri, A., Haynes, K., Bird, D. K., \& Ronan, K. (2018). Bridging the divide between studies on disaster risk reduction education and child-centred disaster risk reduction: a critical review. Children's Geographies, 16(3), 239-251. https://doi.org/10.1080/14733285.2017.1358448
Berroeta, H., Carvalho, L. y Di Masso, A. (2016). Significados del espacio público en contextos de transformación por desastres socionaturales. Revista INVI, 31(87), 143170.https://dx.doi.org/10.4067/S0718-83582016000200005

Bishop, K., \& Corkery, L. (2017). Designing cities with children and young people: beyond playgrounds and skate parks. Abingdon: Routledge.

Borja, J. y Muxi, Z. (2000). El espacio público, ciudad y ciudadanía. Barcelona: Electa.

Bourke, J. (2014). "No Messing Allowed": The Enactment of Childhood in Urban Public Space from the Perspective of the Child. Children. Youth and Environments, 24(1), 25-52. https://doi.org/10.7721/chilyoutenvi.24.1.0025

Brown, C., de Lannoy, A., McCracken, D., Gill, T., Grant, M., Wright, H., \& Williams, S. (2019). Special issue: childfriendly cities. Cities \& Health, 3(1-2), 1-7. https://doi. org/10.1080/23748834.2019.1682836

Campanella, T. (2006). Urban Resilience and the Recovery of New Orleans. Journal of the American Planning Association, 72(1), 141-146.https://doi.org/10.1080/01944360608976734

Carr, A., Abad Merchán, M., \& Ullauri, N. (2020). Conversations about disasters: listening to Ecuadorian children. Children's Geographies, 18(2), 222-233. https://doi.org/10.1080/147 33285.2019.1630712

Churchman, A. (2003). Is There a Place for Children in the City? Journal of Urban Design, 8(2), 99-111. https://doi. org/10.1080/13574800306482

Cele, S., \& van der Burgt, D. (2015). Participation, consultation, confusion: professionals' understandings of children's participation in physical planning. Children's Geographies, 13(1), 14-29.https://doi.org/10.1080/14733285.2013.827873

CEPAL. (2017). Social protection for children at times of disaster. www.cepal.org 
Consejo Nacional de la Infancia. (2016). Estudio Espacios públicos urbanos para niños/as y adolescentes realizado por Estudios y Consultorías Focus Limitada en conjunto con Fundación Patio Vivo. Santiago: Autor.

Derr, V. (2015). Integrating community engagement and children's voices into design and planning education. CoDesign, 11(2), 119-133. https://doi.org/10.1080/15710 $\underline{882.2015 .1054842}$

Derr, V., Corona, Y., \& Gülgönen, T. (2019). Children's Perceptions of and Engagement in Urban Resilience in the United States and Mexico. Journal of Planning Education and Research, 39(1), 7-17. https://doi.org/10.1177/0739456X17723436

Derr, V., Chawla, L., \& Vliet, W. (2017). Children as natural change agents. Child Friendly Cities as Resilient Cities. In K. Bishop and L. Corkery (Eds.), Designing cities with children and young people. Beyond playgrounds and skate parks (pp. 24-35). New York: Routledge.

Driskell, D. (2002). Creating better cities with children and youth: A manual for participation. Paris: UNESCO.

Edmunds, H. (1999). The focus group research handbook. Chicago: NTC/Contemporary Publishing Group.

Fletcher, S., Cox, R., Scannell, L., Heykoop, C., Tobin-Gurley, J., \& Peek, L. (2016). Youth Creating Disaster Recovery and Resilience: A Multi-Site Arts-Based Youth Engagement Research Project. Children, Youth and Environments, 26(1), 148-163. https://doi.org/10.7721/chilyoutenvi.26.1.0148

Freeman, C. (2006). Colliding worlds: planning with children and young people for better cities. In B. Gleeson and N. Sipe (Eds.), Creating child friendly cities: reinstating kids in the city (pp. 69-85). Abingdon: Routledge.

Freeman, C., Henderson, P., \& Kettle, J. (1999). Planning with Children for Better Communities. Bristol: The policy Press.

Freeman, C., \& Tranter, P. (2011). Children and Their Urban Environment: Changing Worlds. Washington DC: Routledge.
French, E., Birchall, J., Landman, K., \& Brown, R. (2019). Designing public open space to support seismic resilience: A systematic review. International Journal of Disaster Risk Reduction, 34, 1-10. https://doi.org/10.1016/j. ijdrr.2018.11.001

Fundación Mi Parque. (2016). Proyecto "Más Plazas para Chile”. https://www.miparque.cl/plaza-la-esperanzaviva-valparaiso/

Fundación Superación de la Pobreza. (2016). Jugando entre riesgos. Representaciones, sentimientos e imágenes de niños y niñas afectados por tres siniestros socioambientales en la región de Valparaíso. Autor.

Gilbert, R., \& O'Brien, C. (2005). Child-and Youth-Friendly Land-Use and TransportPlanning Guidelines. The Ontario Trillium Foundation.

Godschalk, D. (2003). Urban Hazard Mitigation: Creating Resilient Cities. Natural Hazards Review, 4(3). https:// doi.org/10.1061/(ASCE)1527-6988(2003)4:3(136)

Gülgönen, T., \& Corona, Y. (2015). Children's Perspectives on Their Urban Environment and Their Appropriation of Public Spaces in Mexico City. Children, Youth and Environments, 25(2), 208-228. https://doi.org/10.7721/ chilyoutenvi.25.2.0208

Harden, J., Backett-Milburn, K., Scott, S., \& Jackson, S. (2000). Can't Talk, Won't Talk?: Methodological Issues in Researching Children. Sociological Research Online, 5(2), 104-115. https://doi.org/10.5153/sro.486

Instituto Nacional de Estadística. (2017). Síntesis de Resultados Censo 2017. Santiago, Chile: Autor.

Juster, T., Ono, H., \& Stafford, F. (2004). Changing times of American youth: 1981-2003. Michigan: Institute for Social Research University of Michigan.

Koren, D., \& Rus, K. (2019). The potential of open space for enhancing urban seismic resilience: A literature review. Sustainability, 11(21).https://doi.org/10.3390/su11215942 
Middleton, D. (2007). A roof over their heads? The challenge of accommodation following disasters. In Emergency Management Conference, Wellington, New Zealand.

Ministerio de Salud. (2009). Manual de Salud Ambiental Infantil. Santiago: Autor.

Ministerio de Vivienda y Urbanismo. (2015). Comisión de Estudios Habitacionales y Urbanos. "Ciudades con Calidad de Vida: Diagnósticos Estratégicos de Ciudades Chilenas: Sistema Urbano Gran Valparaíso". Santiago: Autor.

Montejano, M., and Moreno, M. (2016). The adaptability of public space in Mexico City after an earthquake: A Preliminary classification. International Journal of Safety and Security Engineering, 6(2), 104-113. https:// doi.org/10.2495/SAFE-V6-N2-104-113

Morgan, M., Gibbs, S., Maxwell, K., \& Britten, N. (2002). Hearing children's voices: methodological issues in conducting focus groups with children aged 7-11 years. Qualitative Research, 2(1), 5-20. https://doi.org/10.1177/14687941 $\underline{02002001636}$

Municipalidad de Valparaíso. (2010). Plan de Desarrollo Comunal de Valparaíso [Pladeco]. Valparaíso: Consultores en Gestión Pública. http://www.munivalpo. cl/transparencia/archivos/plan_desarrollo_comunal/ PLADECO.pdf

Naciones Unidas. (2016). Hábitat III: Conferencia de las Naciones Unidas sobre la Vivienda y el Desarrollo Sostenible. Quito: Autor.

Naciones Unidas. (2015). Memoria. III Conferencia Mundial de Reducción del Riesgo de Desastres, 14 al 18 de marzo 2015, Sendai, Japón.
Peek, L. (2008). Children and Disasters: Understanding Vulnerability, Developing Capacities, and Promoting Resilience - An Introduction. Children, Youth and Environments, 18(1), 1-29. https://www.jstor.org/ stable/10.7721/chilyoutenvi.18.1.0001

Programa de las Naciones Unidas para el Desarrollo. (2014). Plan de Recuperación Post Desastre y Transición al Desarrollo de la comuna de Valparaíso, desde un enfoque participativo y de reducción del riesgo de desastres. Santiago de Chile: Autor.

Ridgers, N., Knowles, Z., \& Sayers, J. (2012). Encouraging play in the natural environment: a child-focused case study of Forest School. Children's Geographies, 10(1), 49-65. https://doi.org/10.1080/14733285.2011.638176

Rubio, M.L. y Santarelli, S. (2011). Lugares rechazados por los adolescentes de la ciudad de Bahía Blanca. Revista Universitaria de Geografía, 20,11-39. https://www.redalyc. org/articulo.oa?id=3832/383239103001

Simpson, B. (1997). Towards the Participation of Children and Young People in Urban Planning and Design. Urban Studies, 34(5-6), 907-925. https://doi.org/10.1080/0042098975880

Sharifi, A., \& Yamagata, Y. (2014). Resilient urban planning: Major principles and criteria. Energy Procedia, 61, 1491-1495. https://doi.org/10.1016/j.egypro.2014.12.154

Tranter, P., \& Malone, K. (2008). Out of Bounds: Insights From Children to Support a Cultural Shift Towards Sustainable and Child-Friendly Cities. Encounter: Education for meaning and social justice, 21(4), 20-26.

Unicef. (2014). For Every Child, A Child-Friendly City. https:// s25924.pcdn.co/wp-content/uploads/2019/10/CFCIBrochure-FINAL-September-2018.pdf

Valentine, G. (2004). Public space and the culture of childhood. London: Routledge. https://doi.org/10.4324/9781315245638 